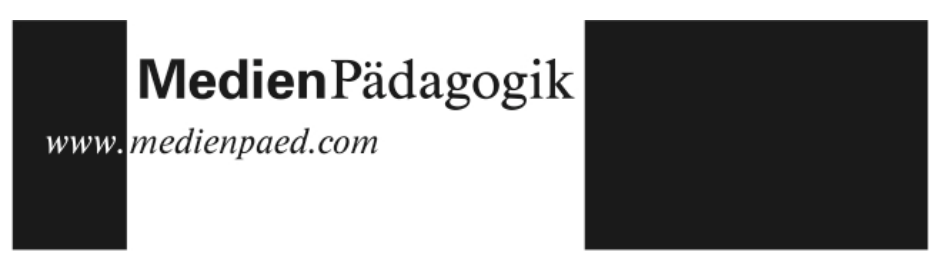

Rezensionen

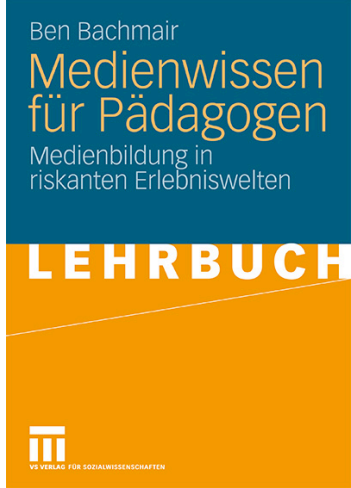

\section{Ben Bachmair}

Medienwissen für Pädagogen

Medienbildung in riskanten Erlebniswelten

Wiesbaden: VS Verlag für Sozialwissenschaften, 2009. 375 Seiten

ISBN: 978-3-531-16305-5

$€ 24.90 ;$ CHF 42.50

\title{
Annäherung an Alltagsästhetik und mobile media als letzte Chance für die Schule?
}

Smartphones als aktuelle technische Plattform für mobile media könnten Schule und das im deutschsprachigen Raum lange tradierte Konzept von «Bildung» endlich fit für die Anforderungen des 21. Jahrhunderts machen so die zentrale These von Ben Bachmair in seinem als «Lehrbuch» gedachten Band Medienwissen für Pädagogen. Das ist zunächst einmal eine steile These des Verfassers. Länger mit dem Thema «Medien und Schule» befasste Leser/innen fühlen sich da schnell an die diversen Medien-Euphorien im Bildungswesen erinnert. Bereits bei der Durchsicht des sehr lesenswerten Bandes wird allerdings schnell deutlich, dass der Autor keineswegs einem untheoretischen und unreflektierten Technik-Fanatismus huldigt. Bereits der Titel, der die erst im Klappentext offenbar werdende starke Orientierung auf mobile media und Web 2.0 noch verschweigt, verweist ja auf ein zunächst noch unscharfes Mehr; «Medienwissen» impliziert etwas anderes als einen neuen, technikinduzierten Hype.

Wie also ist die zentrale These hergeleitet? Der Untertitel Medienbildung in riskanten Erlebniswelten gibt deutliche Hinweise auf die theoretische Einbettung und die Anlage der Argumentationslinie. Mit dem Schlagwort «Medienbildung» bezieht Bachmair in der aktuellen Diskussion um die zentrale theoretische Grundierung der Medienpädagogik deutlich Stellung; das lange dominierende Konzept der "Medienkompetenz» wird damit zwar nicht verabschiedet, steht aber nicht mehr an erster Stelle. An dessen Stelle tritt das bis heute nur im deutschsprachigen Raum (und noch immer nahezu unübersetzbare) einheimische Konstrukt der «Bildung». In enger Anlehnung an Überlegungen von Marotzki entwickelt Bachmair ein Konzept von Bildung, das davon geprägt ist, die aktuellen Medien - und zwar explizit nicht nur in ihren hochkulturellen Nutzungsweisen - zu integrieren. 
Zentral ist dabei die mit vielfältigen Beispielen untermauerte These, dass gerade die in der Schule bisher meist eher negativ konnotierten neuen Medien, wie Web 2.0 und Smartphones, das Potenzial haben, Bildungsprozesse zu initiieren. Dabei - und das ist Bachmair sichtlich ein Anliegen - ermöglichen diese nicht-klassischen Medien insbesondere Jugendlichen, die aus den Bildungsprozessen der Schule auszusteigen drohen oder gar ausgestiegen sind, neue Möglichkeiten der Partizipation.

Ein weiterer, für den Autor zentraler Diskursstrang verbirgt sich hinter dem Adjektiv «riskant». Bachmair spielt damit auf Becks «Risikogesellschaft» und Giddens Überlegungen zu neuen gesellschaftlichen Formen an. Anders als viele Apologeten einer weitreichenden Individualisierung, die endlich die Loslösung von allen Traditionslinien und die freie Verfügbarkeit über die eigene Bastel-Persönlichkeit brächte, fokussiert Bachmair auf die mit diesen Prozessen verbundenen Risiken. Für inn steht außer Frage, dass aus jenen Prozessen der Enttraditionalisierung immer auch Verlierer hervorgehen, die eben gerade angesichts des Individualisierungsparadigmas auf sich selber gestellt sind.

Mit den «Erlebniswelten» wird die dritte zentrale Bezugstheorie deutlich: Schulzes Modell einer Erlebnisgesellschaft, in der die individuelle Erlebnisrationalität zu einem zentralen Motor des Handelns wird. Die Inhalte und Strukturen der neueren Medien (insbesondere in ihren verschiedenen Konvergenzen) sind dann auch - so Bachmair - über weite Strecken daraufhin angelegt, den Rezipienten eine von diesen positiv konnotierte Erlebniswelt zu schaffen: «Die Welt in meinem Sinne». Dass diese aus der Subjektperspektive nachvollziehbare Orientierung gesellschaftlich (mit dem Ziel einer stabilen und partizipativen Demokratie) im Zweifelsfall nicht unproblematisch ist, arbeitet der Verfasser mit einem Rekurs auf Habermas heraus. Womit nicht nur an die Konzepte zur Bildung, sondern auch an die für Baacke zentrale Quelle für die frühen Überlegungen zur Medienkompetenz angeknüpft wird.

Der vierte Theoriestrang bezieht die Perspektive der Medienangebote (und ihren Wandel) selbst ein, die unter dem Label «Alltagsästhetik» im Zusammenhang mit den beobachtbaren Rezeptionsformen analysiert werden. Der zunehmenden Medienkonvergenz wird in diesem Zusammenhang ein besonderer Stellenwert eingeräumt. Mit dem Bezug auf Alltagsästhetik betont Bachmair nochmals, dass es inm keinesfalls nur um die - im schulischen Kontext noch immer dominierende - Rezeption von Artefakten der Hochkultur geht. Vielmehr stehen die alltäglichen, individualisierten und vielfältigen Umgehensweisen mit der gesamten Palette von Medienangeboten im Zentrum. 
Dabei steht für den Autor außer Frage, dass die Medien (und hier insbesondere die Dienste des Web 2.0) längst eigene Kulturräume darstellen, in denen sich die Heranwachsenden mehr oder weniger vielfältig (und damit auch mehr oder weniger kompetent) bewegen.

Die kurze Darstellung der von Bachmair als Ausgangspunkte genutzten Diskurse zeigt eindrücklich, dass das Werk den Anspruch eines «Lehrbuches» auf hohem Niveau ernst nimmt. Dies gilt umso mehr, als der Autor in den fünf Kapiteln noch eine Vielzahl weiterer Verweise zu theoretischen Debatten (z.B. zum aktuellen Stand der Mediendidaktik, zum circuit of culture, aber auch Kritische Theorie etc.), aber auch empirische Befunde zu den jeweiligen Feldern einfügt.

Hilfreich für die Leser/innen ist dabei nicht nur, dass viele der theoretischen Überlegungen anhand von mehr oder weniger ausgearbeiteten Fallstudien aus den Forschungskontexten des Autors besser nachvollziehbar gemacht werden, sondern auch, dass jedes Kapitel mit einer abschließenden Zusammenfassung endet. Hier werden die zentralen Thesen und Begriffe noch einmal in Kurzform aufgenommen, zentrale Definitionen zusammengestellt und um Hinweise für die eigene, vertiefende und weiterführende Lektüre ergänzt.

Hervorzuheben sind m.E. weiterhin die Abschnitte, in denen der Autor den nicht einfachen Anlauf unternimmt, die empirischen Befunde und theoretischen Annahmen für die pädagogische Arbeit nutzbar zu machen, indem mehr oder weniger skizzenhaft einige Konzepte für die Praxis vorgestellt werden. Auch wenn sie sich nicht als schnell kopierbare Vorlagen für den eigenen Unterricht erweisen, liefern diese doch vielfältige und reflektierte Anregungen, die eine Adaption an den eigenen Bedarf und die eigene Praxis ermöglichen.

Kritik an einem solchen Werk zu formulieren ist immer ein wenig wohlfeil, trotzdem seien aus Sicht des Rezensenten einige kritische Fragen gestellt. 
Jenseits einiger auf die Oberfläche zielenden Anmerkungen (nicht immer ist der Text für Anfänger ,leicht' zu lesen) stellen sich beim Blick auf die Tiefenstrukturen einige Fragen:

- Die sehr starke Orientierung auf Individualisierung und Erlebnisorientierung läuft m.E. immer Gefahr, die Ebene der Struktur in problematischem Maße auszublenden. Sowohl die Befunde der PISA-Studien wie auch die im Text vielfach herangezogenen Sinus-Mileus zeigen ja unübersehbar, dass die vor allem in der Herkunftsfamilie vorhandenen Ressourcen wesentlich mit darüber entscheiden, welche Wahlmöglichkeiten überhaupt in den Blick geraten und/oder realisiert werden können. Ein Verweis auf Positionen, die sich kritisch mit der individualisierungstheoretischen Tradition auseinandersetzen könnte im Kontext der Grenzen eines Lehrbuches zumindest andeuten, dass hier auch andere Perspektiven existieren.

- Die Starke Fokussierung auf Web 2.0 und mobile media entspricht zwar dem aktuellen Stand der Medienwelt, läuft aber zum einen Gefahr, die (nach wie vor marktrelevanten) (älteren〉 Medien aus dem Blick zu verlieren. Zum anderen zeigt der Blick auf die Geschichte der je «neuesten〉 Medien, wie schnell sich der Stand der Dinge ändern kann. Was nicht nur hier, sondern in der gesamten medienpädagogischen Debatte m.E. etwas zu kurz kommt, ist die Ausarbeitung von grundlegenden Theorien und Modellen, die eine Analyse von Strukturen jenseits der immer rascheren Folge medientechnischer Hypes und rasanter inhaltlicher Neuerungen ermöglichen.

- Das fünfte Kapitel, in dem exemplarisch einige Analysen von Medien als Kulturprodukten, von medialen Selbstdarstellungen wie auch von Lebenswelt-Ausschnitten vorgestellt werden, fällt gegenüber den vorangegangenen Kapiteln deutlich ab. Die Analysen, die die zentralen Konzepte anhand ihrer Anwendung noch einmal verdeutlichen sollen, bleiben am Ende doch sehr oberflächlich und argumentativ zum Teil schwer nachvollziehbar. Das ist schade, denn die grundsätzliche Idee, auf diesem Weg die vorher theoretisch erarbeiteten Perspektiven noch einmal quasi (praktisch` zu wenden, erscheint durchaus im Sinne eines Lehrbuchs.

Bachmaiers Werk ist, so mein Fazit, für alle lesenswert, die sich in pädagogischen Kontexten mit Medien auseinandersetzen wollen oder müssen. Das gilt - darauf sei explizit hingewiesen - nicht nur für die Novizen, auf die ein 〈Lehrbuch〉 zunächst zielt. Gerade die starke Fokussierung auf aktuelle Entwicklungen der Medienkultur macht das Buch für ein breites Publikum lesenswert. 